\title{
Solar Axions Cannot Explain the XENON1T Excess
}

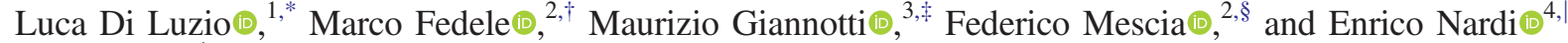 \\ ${ }^{1}$ Deutsches Elektronen-Synchrotron DESY, Notkestraße 85, D-22607 Hamburg, Germany \\ ${ }^{2}$ Department de Física Quàntica i Astrofísica, Institut de Ciències del Cosmos (ICCUB), Universitat de Barcelona, \\ Martí i Franquès 1, E-08028 Barcelona, Spain \\ ${ }^{3}$ Physical Sciences, Barry University, 11300 NE 2nd Avenue, Miami Shores, Florida 33161, USA \\ ${ }^{4}$ INFN, Laboratori Nazionali di Frascati, C.P. 13, 100044 Frascati, Italy
}

(Received 3 July 2020; revised 23 July 2020; accepted 30 July 2020; published 24 September 2020)

\begin{abstract}
We argue that the interpretation in terms of solar axions of the recent XENON1T excess is not tenable when confronted with astrophysical observations of stellar evolution. We discuss the reasons why the emission of a flux of solar axions sufficiently intense to explain the anomalous data would radically alter the distribution of certain type of stars in the color-magnitude diagram in the first place and would also clash with a certain number of other astrophysical observables. Quantitatively, the significance of the discrepancy ranges from $3.3 \sigma$ for the rate of period change of pulsating white dwarfs and exceeds $19 \sigma$ for the $R$ parameter and for $M_{I, \mathrm{TRGB}}$.
\end{abstract}

DOI: 10.1103/PhysRevLett.125.131804

Introduction.-The XENON1T collaboration [1] has reported an excess in low-energy electronic recoil data below $7 \mathrm{keV}$ and peaking around $2-3 \mathrm{keV}$. The collaboration cautions that the excess could be due to an unaccounted background from $\beta$ decays due to a trace amount of tritium, but they also explore the possibility that the signal is due to different types of new physics. The most intriguing interpretation, which also provides the best fit to the data, is given in terms of solar axions, favored over the background-only hypothesis at the $3.5 \sigma$ level. Three production mechanisms contribute to the solar axion flux: (i) atomic recombination and deexcitation, bremsstrahlung, and Compton (ABC) interactions [2] that are controlled by the axion-electron coupling $g_{a e}$, (ii) Primakoff conversion of photons into axions [3] induced by the axion-photon coupling $g_{a \gamma}$, and (iii) axion emission in the M1 nuclear transition of ${ }^{57} \mathrm{Fe}$ [4] that produces monoenergetic $14.4 \mathrm{keV}$ axions and is controlled by and effective axion-nucleon coupling $g_{a n}^{\text {eff }}$. Since this last process cannot play any role in producing events below $10 \mathrm{keV}$, we will not include in our analysis astrophysical observables sensitive to $g_{a n}^{\text {eff }}$. Conversely, axions produced through (i) and (ii) feature a wide spectrum peaking around a few $\mathrm{keV}$. The production rates are independent of the axion mass for $m_{a} \lesssim 100 \mathrm{eV}$. As regards detection, electron recoils occur via the axioelectric effect, which is controlled by $g_{a e}$. Because of this,

Published by the American Physical Society under the terms of the Creative Commons Attribution 4.0 International license. Further distribution of this work must maintain attribution to the author(s) and the published article's title, journal citation, and DOI. Funded by SCOAP ${ }^{3}$. and because the location of the peak around $2-3 \mathrm{keV}$ corresponds roughly to the maximum of the axion energy spectrum for the ABC processes, the Primakoff and ${ }^{57} \mathrm{Fe}$ components are both allowed to be absent as long as there is a nonzero ABC component. This selects $g_{a e}$ as the crucial coupling to attempt to explain the data in terms of the QCD axion [5-9]. Taken at face value, the strength of the XENON1T excess requires $g_{a e} \gtrsim 10^{-12}$, corresponding to an axion decay constant $f_{a} \lesssim 10^{8} \mathrm{GeV}$, and in turn to an axion mass $m_{a} \gtrsim 0.06 \mathrm{eV}$. However, astrophysical considerations indicate that such a large value of $g_{a e}$ is not tenable, as stellar evolution would be drastically affected by the exceedingly large energy losses via axion emission. The strategy that we will follow consists of assuming that $g_{a e}$ and $g_{a \gamma}$ lie in the $90 \%$ C.L. regions resulting from the XENON1T fit [1]. We will then estimate the effects of extra energy losses on a set of astrophysical observables related to red giants branch (RGB) and horizontal branch (HB) stars and to white dwarfs (WDs).

Astrophysical observables and axion couplings.-The axion interactions with photons and electrons read

$$
\mathcal{L}_{\text {int }}=\frac{1}{4} g_{a \gamma} a F_{\mu \nu} \tilde{F}^{\mu \nu}+g_{a e} \frac{\partial_{\mu} a}{2 m_{e}} \bar{e} \gamma^{\mu} \gamma_{5} e,
$$

where the couplings can be related to model-dependent dimensionless coefficients as $g_{a \gamma}=(\alpha / 2 \pi)\left(C_{a \gamma} / f_{a}\right)$ and $g_{a e}=C_{a e}\left(m_{e} / f_{a}\right)$. In benchmark axion models $C_{a \gamma}$ and $C_{a e}$ are typically of $\mathcal{O}(1)$, although strong enhancements and/or suppressions are possible in specific cases [10-14]. In the following, we will adopt the notation $g_{\gamma 10} \equiv g_{a \gamma} \times$ $\left(10^{10} \mathrm{GeV}\right)$ and $g_{e 13} \equiv g_{a e} \times 10^{13}$. Axions with couplings as large as $g_{e 13} \sim 10, g_{\gamma 10} \sim 1$ would be abundantly 
produced in several types of stars without being trapped, and thus would efficiently drain energy from the star cores.

Astrophysical considerations have been systematically used to place severe bounds on light, weakly interacting particles, such as neutrinos and axions [15]. Noticeably, a set of anomalous observations have recently led to speculations that new physics is at play $[14,16,17]$, and the axion case appears especially compelling $[18,19]$. The most effective observables to constrain $g_{a e}$ and $g_{a \gamma}$ are described below.

Tip of RGB stars in globular cluster: We denote by $M_{I, \mathrm{TRGB}}$ the luminosity of the tip of the RGB in globular clusters. RG stars are characterized by a He core and a burning $\mathrm{H}$ shell. During the RGB evolution, the ashes from the burning shell increase the He-core mass, while the star luminosity (determined by equilibrium at the surface of the He core between thermal pressure supporting the nondegenerate envelope against the gravity pull from the core) keeps growing. The process continues until the core reaches sufficiently large temperatures and densities $\left(T \sim 10^{8} \mathrm{~K}, \rho=10^{6} \mathrm{~g} \mathrm{~cm}^{-3}\right)$ to ignite $\mathrm{He}$, an event known as the He flash. At this stage, the star has reached its maximum luminosity $M_{I, \mathrm{TRGB}}$, after which it shrinks and moves to the HB. If an additional core-cooling mechanism were at play, He ignition would be delayed, the core would accrete a larger mass, and the star would reach higher luminosities. Therefore, measurements of $M_{I, \mathrm{TRGB}}$ allow us to test the rate of cooling during the RGB phase. The method is particularly effective for constraining $g_{a e}$ since, in $\mathrm{RG}$ cores, axions can be efficiently produced via electron bremsstrahlung. The most recent analyses [20-22] have derived comparable constraints. Here we adopt the result of the analysis of the Large Magellanic Cloud in Refs. [23,24], which provides the most conservative bound $M_{I, \mathrm{TRGB}}=-4.047 \pm 0.045 \mathrm{mag}$. In terms of $g_{a e}$, this observable can be written as $[20,25]$

$$
\begin{aligned}
M_{I, \mathrm{TRGB}}^{\text {theo }}= & -4.08 \\
& -0.25\left(\sqrt{g_{e 13}^{2}+0.93}-0.96-0.17 g_{e 13}^{1.5}\right),
\end{aligned}
$$

which results from an analytic fit to ten evolutionary track points reaching close to the RGB tip obtained from numerical simulations [26] and corresponding to values of $g_{e 13}$ up to 9 [20]. The associated theoretical uncertainty is $\sigma^{2}=0.039^{2}+\left(0.046+0.012 g_{e 13}\right)^{2}$ [20].

$R$ parameter: After He ignition, the RG core expands and its density decreases by 2 orders of magnitude. The star migrates to the $\mathrm{HB}$ and remains supported by He burning in a nondegenerate core. The ratio $R=N_{\mathrm{HB}} / N_{\mathrm{RGB}}$ between the number of stars in globular clusters in the $\mathrm{HB}$ and in the upper portion of the RGB directly measures the duration of He burning in the HB phase. The value $R=1.39 \pm 0.03$ was obtained in Ref. [27] from the analysis of 39 clusters. The duration of the HB phase can be affected by $g_{a e}$-related processes both directly and indirectly. If $g_{a e}$ is sufficiently large, axion emission would directly produce extra cooling of the He core. The star self-regulates by slightly contracting and the core temperature increases, speeding up the $\mathrm{He}$ burning rate. Once the He fuel is exhausted, the star turns into a WD. The indirect effect is related to the growth of the degenerate He core during the RGB phase previously discussed. HB stars would unavoidably inherit a more massive core from the parent RGs, resulting in an increased He burning rate to contrast the larger gravitational pull and shortening further the duration of the HB phase. Note that the indirect effect of $g_{a e}$ is so important that, for $g_{e 13} \sim 15$, it would suffice to depopulate almost completely the HB in the color-magnitude diagram (CMD) $(R \approx 0)$. Cooling of HB stars can also proceed via the Primakoff effect $\propto g_{a \gamma}^{2}$, which is particularly efficient at the typical temperatures and densities of HB cores $\left(T \sim 10^{8} \mathrm{~K}, \rho=10^{4} \mathrm{~g} \mathrm{~cm}^{-3}\right)$. For sufficiently large values of $g_{a \gamma}, R$ can still decrease well below the observed values even when $g_{a e} \approx 0$. Hence, an accurate determination of this observable allows us to probe the axion coupling to both photons and electrons. In terms of $g_{a e}$ and $g_{a \gamma}$, the $R$ parameter can be written as $[19,28]$

$$
\begin{aligned}
R^{\text {theo }}= & 7.33 Y-0.095 \sqrt{21.86+21.08 g_{\gamma 10}} \\
& +0.02-1.61 \delta \mathcal{M}_{c}-0.005 g_{e 13}^{2}, \\
\delta \mathcal{M}_{c}= & 0.024\left(\sqrt{g_{e 13}^{2}+1.23^{2}}-1.23-0.138 g_{e 13}^{1.5}\right),
\end{aligned}
$$

where $\delta \mathcal{M}_{c}$ is the change in the He-core mass, and $Y \simeq$ $0.255 \pm 0.002$ is the primordial He abundance. The relative errors on $\delta \mathcal{M}_{c}$, which represents the main theoretical uncertainty from astrophysics. and the one on $Y$, are of the same order. Hence, due to the larger coefficient multiplying $Y$, the uncertainty from $\delta \mathcal{M}_{c}$ can be neglected. Similar to Eq. (2), this expression is derived from an analytic fit to evolutionary track points calculated with stellar evolutionary codes modified to account for axion emission $[19,28]$; thus, it is quantitatively reliable up to values of $g_{a e}$ not much larger than those corresponding to the last point fitted (for definiteness $g_{e 13} \sim 9$ ). Thus, we will not input into these expressions the much larger XENON1T values $g_{e 13} \sim 30$. Rather, very conservatively, we will limit ourselves to estimate the tension between the observed values of $M_{I, \mathrm{TRGB}}$ and $R$, and the values resulting from Eqs. (2) and (3) when evaluated at $g_{e 13} \sim 9\left(g_{a \gamma} \approx 0\right)$. As regards values of $g_{a \gamma}$ too large to be used in Eq. (3), they can be directly constrained from the lifetime of HB stars which, in the presence of extra cooling, scales as $\sim L_{0} /\left(L_{0}+L_{a}\right)$ with $L_{0}\left(L_{a}\right)$ the standard (axion) core luminosity [15]. Hence, for $g_{\gamma 10} \gtrsim 1$, rather than Eq. (3), we will use

$$
R^{\text {theo }} \approx \frac{a^{2}}{a+b g_{\gamma 10}^{2}},
$$


TABLE I. Measured values of astrophysic observables and expected ranges, for $g_{a e}, g_{a \gamma}$ falling within the $1 \sigma$ region of the XENON1T fit $\left(\bar{g}_{e 13} \in[28,35]\right)$. $\dot{\Pi}_{\mathrm{WD} i}$ are in units of $\left[10^{-15} \mathrm{~s} / \mathrm{s}\right]$. For $R$ and $M_{I, \mathrm{TRGB}}$ the expected regions and tensions correspond to $g_{e 13}=\bar{g}_{e 13}\left(g_{a \gamma}=0\right) \geq 9$ (see text).

\begin{tabular}{lccc}
\hline \hline Observable & Measured & Expected & Tension \\
\hline$R$ parameter & $1.39 \pm 0.03$ & $\leq 0.83\left(g_{e 13}=9\right)$ & $19 \sigma^{\star}$ \\
$M_{I, \text { TRGB }}^{\mathrm{LMC}}(\mathrm{mag})$ & $-4.047 \pm 0.045$ & $\leq-4.92\left(g_{e 13}=9\right)$ & $19 \sigma^{\star}$ \\
$g_{e 13}^{\text {WDLF }}$ & $\leq 2.8(3 \sigma)$ & $29.7 \pm 4.8$ & $5.6 \sigma$ \\
$\dot{\Pi}_{\mathrm{L} 19-2}^{(113)}$ & $3.0 \pm 0.6$ & $57 \pm 16$ & $3.4 \sigma$ \\
$\dot{\Pi}_{\mathrm{L} 19-2}^{(192)}$ & $3.0 \pm 0.6$ & $95 \pm 27$ & $3.4 \sigma$ \\
$\dot{\Pi}_{\mathrm{PG} 1351+489}$ & $200 \pm 90$ & $19620 \pm 5730$ & $3.4 \sigma$ \\
$\dot{\Pi}_{\mathrm{G} 117-\mathrm{B} 15 \mathrm{~A}}$ & $4.2 \pm 0.7$ & $113 \pm 33$ & $3.3 \sigma$ \\
$\dot{\Pi}_{\mathrm{R} 548}$ & $3.3 \pm 1.1$ & $87 \pm 25$ & $3.3 \sigma$ \\
\hline \hline
\end{tabular}

with $a=(6.26 Y-0.12)$ and $b=0.41$ [27]. Note that Eq. (4) neglects both direct and indirect effects of $g_{a e}$ on $\mathrm{HB}$ and RGB stars, and hence it would also yield conservative limits.

White dwarf luminosity function: The third observable we consider is the distribution of WDs as a function of their luminosity (WDLF). The WDLF measures the WD cooling efficiency, and thus allows us to place strong bounds on new exotic cooling processes, including axion emission (see Ref. [29] for a review). WDs are compact objects whose hydrostatic equilibrium is supported by electron degeneracy pressure; hence, axion emission from WDs would dominantly depend on $g_{a e}$. Here we will use the bound $g_{e 13}^{\mathrm{WDF}} \leq 2.8$ obtained in Ref. [30].

Rate of period change of WD variables: WD variables (WDVs) are WDs whose luminosity varies periodically,

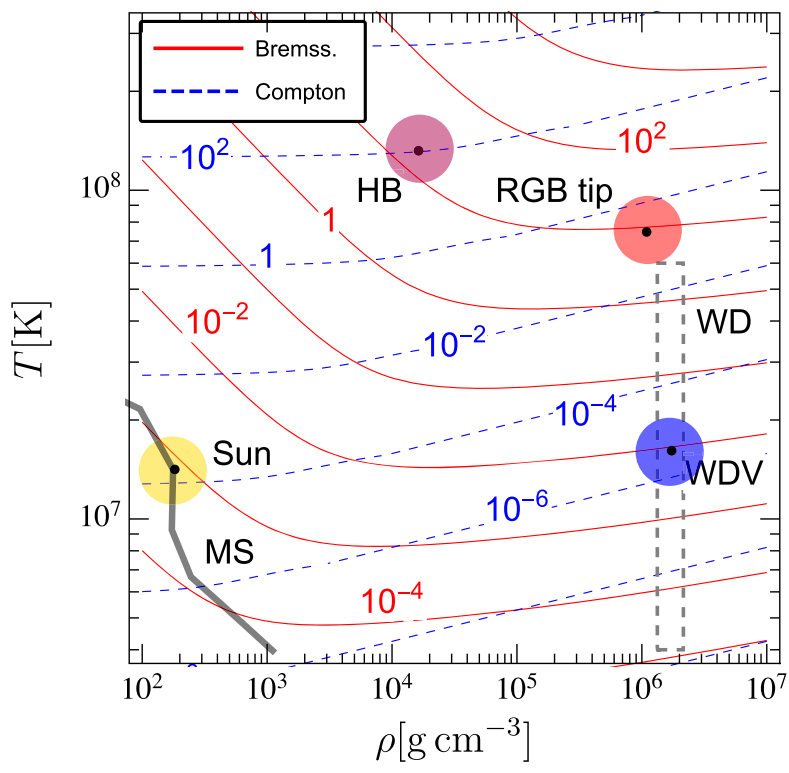

FIG. 1. Contours of the axion energy-loss rates per unit mass, in erg $\mathrm{g}^{-1} \mathrm{~s}^{-1}$, for a pure He plasma and $g_{e 13}=4.3$. with a period $\Pi$ ranging from a few to several minutes. Because the oscillation period depends on the luminosity, a secular change of the period $\dot{\Pi}$ tracks the rate of decrease of the star luminosity. To a very good approximation $\dot{\Pi} / \Pi$ is proportional to the cooling rate $\dot{T} / T$; hence. a measurement of $\Pi$ allows us to constrain possible sources of extra cooling (see Ref. [31] for a review). Here we consider four WDVs: G117-B15A [32], R548 [33], L19-2 [34] (for two pulsation modes), and PG1351 + 489 [35]. We list in Table I the corresponding measured values of $\dot{\Pi} / \Pi$. Theoretically, the rate of change in the WD pulsating period as a function of $g_{e 13}$ can be parametrized as [18] $\dot{\Pi}_{\mathrm{W} D_{i}}^{\text {theo }}=a_{i}+b_{i} g_{e 13}^{2}$, where $a_{i}$ and $b_{i}$ are parameters specific for each WD.

XENONIT vs astrophysics. - Figure 1 shows contours of the axion energy-loss rates per unit mass in a temperature vs density plane, for a pure He plasma. Contour isolines for energy loss due to Compton (dashed blue) and bremsstrahlung (solid red) processes, which are controlled by $g_{a e}$, are also shown. For reference, we have fixed $g_{e 13}=4.3$, which corresponds to the RGB bound from M5 [20]. Energy-loss rates for different values of $g_{a e}$ can be easily obtained, recalling that they scale as $g_{a e}^{2}$. The labeled disks in the figure show the position of the RGB tip and of a typical HB star (of mass $0.8 M_{\odot}$ ) and a range of WDs with luminosities varying from $5 \times 10^{-4}$ to $5 \times 10^{-1} L_{\odot}$ (dashed gray rectangle). The blue disk indicates the temperature and density of a typical WD variable [31]. The location of the Sun is marked with a yellow disk on top of the broken gray line, which locates main sequence (MS) stars of different masses. Note that, since MS stars, including the Sun, are supported by $\mathrm{H}$ burning cores, their position with respect to the energy-loss isolines for the He plasma is approximate and slightly shifted toward larger rates. The picture shows clearly that the Sun is a relatively faint axion emitter with respect to other stellar objects, so that values $g_{e 13} \gtrsim 10$ as required to account for the XENON1T excess would unavoidably turn other stars into bright "axion lighthouses." The RGB would extend to higher luminosities 
than the ones observed, and the decreased duration of the He burning phase would depopulate the HB, to the point that, for smaller clusters with relatively few stars, already for $g_{e 13} \sim 15$ we would expect $R \approx 0$. In short, regardless of other details, a value $g_{e 13} \sim 30$ would definitely destroy the agreement between stellar evolution models and the observed CMD.

Quantifying the tension.-The projections of the XENON1T 90\% C.L. best fit region onto the $\left(g_{a e}, g_{a \gamma}\right)$, $\left(g_{a e}, g_{a n}^{\text {eff }}\right)$, and $\left(g_{a e} g_{a \gamma}, g_{a e} g_{a n}^{\text {eff }}\right)$ planes are given in Fig. 8 of Ref. [1]. Since only $g_{a e}$ and $g_{a \gamma}$ can be responsible for the anomalous XENON1T data below $7 \mathrm{keV}$, we focus on the best fit region for these two couplings, which corresponds the blue band in Fig. 2. In the figure we also show the $2 \sigma$ limits on $g_{a e}, g_{a y}$ obtained from each single astrophysical observable, as well as the result of a global fit to the entire set of stellar cooling data. The curve depicting the CAST [36] limit in the $\left(g_{a e}, g_{a \gamma}\right)$ plane in Ref. [1] was taken from Ref. [37]. We update this bound with the most recent CAST results [38] which, in the $g_{a e} \simeq 0$ limit, and for $m_{a} \lesssim 20 \mathrm{meV}\left(m_{a} \lesssim 0.7 \mathrm{eV}\right)$, correspond to $g_{a \gamma}<0.66(2.0) \times 10^{-10} \mathrm{GeV}^{-1}$. These limits are represented in Fig. 2 by the two green lines, in which we have folded in the effects of a nonzero $g_{a e}$ that would increase the production of solar axions and strengthen the bounds. The vertical dashed line is the LUX limit [39]. The gray horizontal line at $g_{\gamma 10}=4.1$ corresponds to the limit from a global fit to solar data, which includes the measured flux of ${ }^{8} \mathrm{~B}$ and ${ }^{7} \mathrm{Be}$ neutrinos as well as additional data

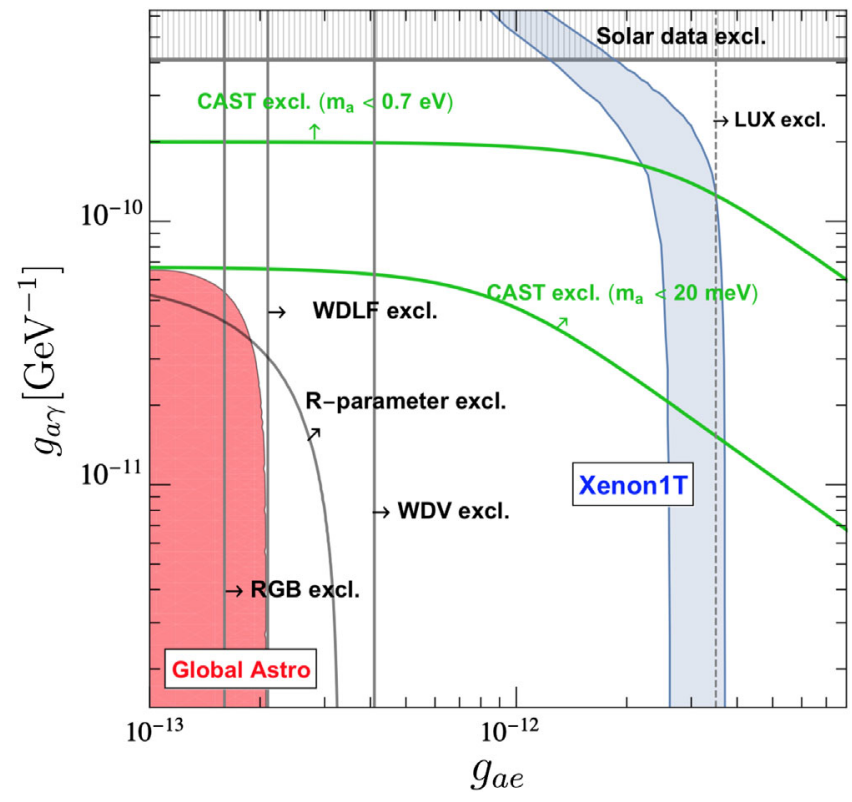

FIG. 2. XENON1T 90\% C.L. fit (blue region). $3 \sigma$ exclusion limit from solar data (gray hatched region). $2 \sigma$ LUX limit (gray dashed line) and CAST limits for $m_{a}<20 \mathrm{meV}$ and $m_{a}<0.7 \mathrm{eV}$ (green lines). Individual $2 \sigma$ limits from $R$ parameter, TRGB, WDLF, WDVs (gray lines), and $2 \sigma$ global bound from astrophysics (red region). inferred from helioseismology observations [40]. This is about a factor of 2 stronger than the bound labeled "solar $\nu$ " in the upper panel of Fig. 8 in Ref. [1], which is taken from Ref. [41] (see [42]). To assess quantitatively the discrepancy between the values of $g_{a e}$ and $g_{a y}$ needed to reproduce the XENON1T excess, we proceed as follows: we first extract the allowed ranges from the 90\% C.L. region of Ref. [1] not excluded by solar data (the blue area in Fig. 2). This region can be parametrized by means of an effective coupling [14]

$$
\bar{g}_{e 13}^{4}=g_{e 13}^{2}\left(g_{e 13}^{2}+200 g_{\gamma 10}^{2}\right) .
$$

The $90 \%$ C.L. (68\% C.L.) region of XENON1T is then well represented by the range $\bar{g}_{e 13} \in[26,37]\left(\bar{g}_{e 13} \in[28,35]\right)$. Varying $g_{a e}$ and $g_{a \gamma}$ with the constraint that $\bar{g}_{e 13}$ remains within this range, we estimate the range of values for the astrophysical observables implied by the XENON1T data, and we confront them with the measured values. Our results are collected in Table I. For each observable, the tension given in the fourth column is evaluated by dividing the difference between the value implied by the XENON1T data and the astrophysical determination by the total uncertainty. Given that the statistical distributions are at best only approximately known, these results are only indicative and have no rigorous Gaussian meaning. It is apparent that the large $g_{a e}$ required to fit the XENON1T excess are in strong conflict with all the astrophysical observables. The discrepancy is at the level of $\sim 3.4 \sigma$ for the WDVs cooling rates (last five rows in the Table) and reaches $\sim 6 \sigma$ for the WDLF in the third row. As regards the first two rows, the expected values of $R^{\text {theo }}$ and of $M_{I, T R G B}^{\text {theo }}$ reported in the table are obtained, respectively, from Eqs. (3) and (2) by setting $g_{e 13}=9$, rather than by inserting the much larger values $g_{e 13} \sim 30$ needed to account for the XENON1T data. This is a precautionary procedure that we have adopted to avoid extrapolating Eqs. (2) and (3) to values of $g_{a e}$, for which the quantitative accuracy of these parametrizations cannot be easily assessed. We have then marked with a $\star$ the corresponding tensions. We expect that values of the observables in agreement with the XENON1T solar axion fit would result in much larger tensions. For example, already for $g_{e 13} \approx 15$ Eq. (3) would yield $R \approx 0$, corresponding to a complete depopulation of the HB, and $46 \sigma$ away from observations.

Conclusions.-In this Letter, we have explained why astrophysical observations firmly exclude that solar axions could account for the XENON1T excess. Other explanations based on solar production of new light particles or on modifications of neutrino properties (such as a neutrino magnetic moment) are also prone to severe astrophysical constraints, and as long as the corresponding new physics processes would also occur in RG, HB, and WD stellar cores, they can likewise be excluded [43]. 
If it will be eventually found that the tritium background or other systematic effects $[45,46]$ are not responsible for the excess, other mechanisms involving either absorption or scattering of new particles of nonsolar origin off target electrons [47-51], although less compelling than the QCD axion, might still provide viable explanations for the XENON1T data.

We thank Axel Lindner for useful comments. L. D. L. is supported by the Marie Skłodowska-Curie Individual Fellowship Grant AXIONRUSH (GA 840791). M. F. and F. M. are supported by MINECO Grant No. FPA201676005-C2-1-P, Maria de Maetzu program Grants No. MDM-2014-0367 of ICCUB and No. 2017 SGR 929. E. N. is supported by the INFN Iniziativa Specifica, Theoretical Astroparticle Physics (TAsP).

Note added.-Recently, Refs. [52,53] appeared claiming that, besides the axioelectric effect, also the inverse Primakoff process can contribute to the detection of solar axions by XENON1T. This would relax the best fit region toward lower values of $g_{a e}$ at the cost of increasing $g_{a \gamma}$. This can relax the tension with astrophysical bounds; however, using the results of Ref. [52,53] we have verified that the discrepancy with the $R$ parameter remains at least at the level of $8 \sigma$.

*luca.diluzio@desy.de

†marco.fedele@icc.ub.edu

*MGiannotti@barry.edu

§mescia@ub.edu

"enrico.nardi@lnf.infn.it

[1] E. Aprile et al. (XENON Collaboration), arXiv:2006.09721.

[2] J. Redondo, J. Cosmol. Astropart. Phys. 12 (2013) 008.

[3] H. Primakoff, Phys. Rev. 81, 899 (1951).

[4] S. Moriyama, Phys. Rev. Lett. 75, 3222 (1995).

[5] R. D. Peccei and H. R. Quinn, Phys. Rev. Lett. 38, 1440 (1977).

[6] R. D. Peccei and H. R. Quinn, Phys. Rev. D 16, 1791 (1977).

[7] S. Weinberg, Phys. Rev. Lett. 40, 223 (1978).

[8] F. Wilczek, Phys. Rev. Lett. 40, 279 (1978).

[9] Our results apply also to explanations based on generic axionlike particles, for which there is no theoretical relation between $m_{a}$ and the coupling strengths, and that are unrelated to the strong $C P$ problem.

[10] L. Di Luzio, F. Mescia, and E. Nardi, Phys. Rev. Lett. 118, 031801 (2017).

[11] L. Di Luzio, F. Mescia, and E. Nardi, Phys. Rev. D 96, 075003 (2017).

[12] L. Di Luzio, F. Mescia, E. Nardi, P. Panci, and R. Ziegler, Phys. Rev. Lett. 120, 261803 (2018).

[13] F. Bjorkeroth, L. Di Luzio, F. Mescia, E. Nardi, P. Panci, and R. Ziegler, Phys. Rev. D 101, 035027 (2020).

[14] L. Di Luzio, M. Giannotti, E. Nardi, and L. Visinelli, arXiv:2003.01100.
[15] G. Raffelt, Stars as Laboratories for Fundamental Physics: The Astrophysics of Neutrinos, Axions, and Other Weakly Interacting Particles (University of Chicago Press, Chicago, 1996).

[16] S. Hoof, F. Kahlhoefer, P. Scott, C. Weniger, and M. White, J. High Energy Phys. 03 (2019) 191; 11 (2019) 099(E).

[17] P. Di Vecchia, M. Giannotti, M. Lattanzi, and A. Lindner, Proc. Sci., Confinement2018 (2019) 034.

[18] M. Giannotti, I. G. Irastorza, J. Redondo, A. Ringwald, and K. Saikawa, J. Cosmol. Astropart. Phys. 10 (2017) 010.

[19] M. Giannotti, I. Irastorza, J. Redondo, and A. Ringwald, J. Cosmol. Astropart. Phys. 05 (2016) 057.

[20] N. Viaux, M. Catelan, P. B. Stetson, G. G. Raffelt, J. Redondo, A. A. R. Valcarce, and A. Weiss, Phys. Rev. Lett. 111, 231301 (2013).

[21] O. Straniero, I. Dominguez, M. Giannotti, and A. Mirizzi, in 13th Patras Workshop on Axions, WIMPs and WISPs (2018), pp. 172-176 [arXiv:1802.10357].

[22] S. A. Díaz, K.-P. Schröder, K. Zuber, D. Jack, and E. E. B. Barrios, arXiv:1910.10568.

[23] W. L. Freedman et al., Astrophys. J. 882, 34 (2019).

[24] W. L. Freedman, B. F. Madore, T. Hoyt, I. S. Jang, R. Beaton, M. G. Lee, A. Monson, J. Neeley, and J. Rich, Astrophys. J. 891, 57 (2020).

[25] F. Capozzi and G. Raffelt, arXiv:2007.03694.

[26] N. Viaux, M. Catelan, P. B. Stetson, G. Raffelt, J. Redondo, A. A. R. Valcarce, and A. Weiss, Astron. Astrophys. 558, A12 (2013).

[27] A. Ayala, I. Dominguez, M. Giannotti, A. Mirizzi, and O. Straniero, Phys. Rev. Lett. 113, 191302 (2014).

[28] O. Straniero, A. Ayala, M. Giannotti, A. Mirizzi, and I. Dominguez, in 11th Patras Workshop on Axions, WIMPs and WISPs (2015), pp. 77-81, https://dx.doi.org/10.3204/ DESY-PROC-2015-02/straniero_oscar.

[29] J. Isern, in IAU Symposium 357: White Dwarfs as Probes of Fundamental Physics and Tracers of Planetary, Stellar \& Galactic Evolution (2020) [arXiv:2002.08069].

[30] M. M. M. Bertolami, B. E. Melendez, L. G. Althaus, and J. Isern, J. Cosmol. Astropart. Phys. 10 (2014) 069.

[31] A. H. Córsico, L. G. Althaus, M. M. M. Bertolami, and S. Kepler, Astron. Astrophys. Rev. 27, 7 (2019).

[32] A. H. Corsico, L. G. Althaus, M. M. Bertolami, A. D. Romero, E. Garcia-Berro, J. Isern, and S. Kepler, Mon. Not. R. Astron. Soc. 424, 2792 (2012).

[33] A. Corsico, L. Althaus, A. Romero, A. Mukadam, E. Garcia-Berro, J. Isern, S. Kepler, and M. Corti, J. Cosmol. Astropart. Phys. 12 (2012) 010.

[34] A. H. Córsico, A. D. Romero, L. G. Althaus, E. GarcíaBerro, J. Isern, S. Kepler, M. M. M. Bertolami, D. J. Sullivan, and P. Chote, J. Cosmol. Astropart. Phys. 07 (2016) 036.

[35] T. Battich, A. H. Córsico, L. G. Althaus, M. M. M. Bertolami, and M. Bertolami, J. Cosmol. Astropart. Phys. 08 (2016) 062.

[36] S. Andriamonje et al. (CAST Collaboration), J. Cosmol. Astropart. Phys. 12 (2009) 002.

[37] K. Barth et al., J. Cosmol. Astropart. Phys. 05 (2013) 010.

[38] V. Anastassopoulos et al. (CAST Collaboration), Nat. Phys. 13, 584 (2017). 
[39] D. Akerib et al. (LUX Collaboration), Phys. Rev. Lett. 118, 261301 (2017).

[40] N. Vinyoles, A. Serenelli, F. L. Villante, S. Basu, J. Redondo, and J. Isern, J. Cosmol. Astropart. Phys. 10 (2015) 015.

[41] P. Gondolo and G. G. Raffelt, Phys. Rev. D 79, 107301 (2009).

[42] For values of the couplings allowed by astrophysics the solar axion luminosity $L_{a}$ is a negligible fraction of the total luminosity, for example, $L_{a} \approx 1.85 \times 10^{-3} g_{\gamma 10}^{2} L_{\odot}$ for Primakoff emission [41]. Hence, effects on the Sun lifetime are also negligible.

[43] Astrophysical constraints could only be evaded in exotic models in which the couplings strongly depend on the stellar environment, like the core density and temperature, see, e.g., [44].

[44] J. Redondo, Can the PVLAS particle be compatible with the astrophysical bounds? Ph. D. thesis, Barcelona, Autonoma University, 2007.
[45] C. Dessert, J. W. Foster, Y. Kahn, and B. R. Safdi, arXiv:2006.16220.

[46] M. Szydagis, C. Levy, G. Blockinger, A. Kamaha, N. Parveen, and G. Rischbieter, arXiv:2007.00528.

[47] F. Takahashi, M. Yamada, and W. Yin, arXiv:2006.10035.

[48] K. Kannike, M. Raidal, H. Veermäe, A. Strumia, and D. Teresi, arXiv:2006.10735.

[49] G. Alonso-Álvarez, F. Ertas, J. Jaeckel, F. Kahlhoefer, and L. Thormaehlen, arXiv:2006.11243.

[50] C. Boehm, D. G. Cerdeno, M. Fairbairn, P. A. Machado, and A. C. Vincent, arXiv:2006.11250.

[51] B. Fornal, P. Sandick, J. Shu, M. Su, and Y. Zhao, arXiv:2006.11264.

[52] C. Gao, J. Liu, L.-T. Wang, X.-P. Wang, W. Xue, and Y.-M. Zhong, this issue, Phys. Rev. Lett. 125, 131806 (2020).

[53] J. B. Dent, B. Dutta, J. L. Newstead, and A. Thompson, following Letter, Phys. Rev. Lett. 125, 131805 (2020). 